\title{
High signal-to-noise ratio Q-switching erbium doped fiber laser pulse emission utilizing single layer trivial transfer graphene film saturable absorber
}

\begin{abstract}
This paper presents the high signal-to-noise ratio (SNR) Q-switched erbium-doped fiber laser pulse emission using a commercial single layer graphene (SLG) film as a saturable absorber (SA). A sandwiched-type structure with transferred single layer SLG film between two fiber ferrules is formed to function as the SA. Q-switched pulse emission with repetition rate from $47.25 \mathrm{kHz}$ to $67.39 \mathrm{kHz}$ and round-trip time per oscillation from $7.42 \mu \mathrm{s}$ to $10.36 \mu \mathrm{s}$ are obtained from the laser cavity set-up. The SNR of $62.64 \mathrm{~dB}$ shows a good quality of pulse generation using the SLG film as SA. The effortless production of SLG is enabling factor to produce fast fabrication and low cost SA for application in Q-switched pulsed fiber lasers.
\end{abstract}

Keyword: Erbium-doped fiber laser; Q-switched pulsed laser; Saturable absorber; Signal to noise ratio; Single layer graphene film 